\title{
Long-Term Effects of Permanent Vestibular Lesions on Hippocampal Spatial Firing
}

\author{
Noah A. Russell, ${ }^{1}$ Arata Horii, ${ }^{2,3}$ Paul F. Smith, ${ }^{2}$ Cynthia L. Darlington, ${ }^{1,2}$ and David K. Bilkey ${ }^{1}$ \\ ${ }^{1}$ Department of Psychology and Neuroscience Research Centre and 2Department of Pharmacology and Toxicology, School of Medical Sciences, University of \\ Otago, Dunedin, 9001 New Zealand, and ${ }^{3}$ Department of Otolaryngology, Osaka University Medical School, Osaka, 565-0871 Japan
}

The hippocampus is thought to be important for spatial representation processes that depend on the integration of both self-movement and allocentric cues. The vestibular system is a particularly important source of self-movement information that may contribute to this spatial representation. To test the hypothesis that the vestibular system provides self-movement information to the hippocampus, rats were given either a bilateral labyrinthectomy $(n=6)$ or a sham surgery $(n=6)$, and at least $60 \mathrm{~d}$ after surgery hippocampal CA1 neurons were recorded extracellularly while the animals foraged freely in an open arena. Recorded cells were classified as complex spiking $(n=$ $80)$ or noncomplex spiking $(n=33)$ neurons, and their spatial firing fields (place fields) were examined. The most striking effect of the lesion was that it appeared to completely abolish location-related firing. The results of this and previous studies provide converging evidence demonstrating that vestibular information is processed by the hippocampus. The disruption of the vestibular input to the hippocampus may interfere with the reconciliation of internal self-movement signals with the changes to the external sensory inputs that occur as a result of that movement. This would disrupt the ability of the animal to integrate allocentric and egocentric information into a coherent representation of space.

Key words: hippocampus; vestibular; labyrinthectomy; place cell; place field; self-motion; compensation; navigation

\section{Introduction}

To navigate successfully in a complex world, organisms require a representation of the spatial environment. The hippocampus appears to have a role in this process (O'Keefe and Nadel, 1978) because hippocampal "place cells" encode the animal's location (O’Keefe and Dostrovsky, 1971). Each place cell will usually fire in a subregion of the environment (its "place field"), which is generally stable across many recording sessions (Muller et al., 1987). Place cell firing appears to depend on the integration of self-motion and external cues (Whishaw, 1998). For example, the position of place fields can be controlled by visual cues, and they become less stable in the dark (Markus et al., 1994), presumably because of cumulative self-motion errors. Sources of self-motion information include sensory flow, motor efferent copy, and vestibular inputs. The vestibular system is particularly important because it directly senses angular and linear accelerations of the head (for review, see Wilson and Melvill Jones, 1979).

Several previous studies have provided evidence for vestibulohippocampal information flow (for review, see Smith, 1997). Rotation and disorientation affect both place fields and hippocampal electroencephalographic activity (O'Mara et al., 1994; Sharp et al., 1995; Wiener et al., 1995; Dudchenko et al., 1997; Gavrilov

\footnotetext{
Received Jan. 8, 2003; revised May 5, 2003; accepted May 7, 2003.

This work was supported by The Marsden Fund of New Zealand (D.B.) and The New Zealand Neurological Foundation Project Grant (703) (P.S., C.D.). We thank Ping Liu for her assistance with surgeries.

Correspondence should be addressed to Dr. David K. Bilkey, Department of Psychology and Neuroscience Research Centre, University of Otago, 95 Union Street, P.0. Box 56, Dunedin, 9001 New Zealand. E-mail: sycodkb@psy.otago.ac.nz.

Copyright $\odot 2003$ Society for Neuroscience $\quad$ 0270-6474/03/236490-09\$15.00/0
}

et al., 1998). Electrical stimulation of the vestibular labyrinth or medial vestibular nucleus alters electrophysiological activity in the hippocampus (Cuthbert et al., 2000), and transient inactivation of the peripheral vestibular apparatus disrupts place fields (Stackman et al., 2002). Furthermore, activation or inactivation of the vestibular system produces specific neurochemical changes in the hippocampus (Zheng et al., 2001). None of these previous studies, however, have determined the long-term effects of bilateral vestibular deafferentation on the hippocampal spatial representation.

The present study investigated the role of the vestibular system in hippocampal function by using a permanent lesion approach in which the vestibular receptors are damaged directly and completely, because previous studies have shown that intratympanic injections of ototoxins can result in incomplete lesions (Jensen, 1983). Bilateral labyrinthectomy reduces neuronal activity in both vestibular nuclei equally and produces static and dynamic (motion-dependent) symptoms. Although the precise time course of the compensation of these symptoms has not been quantified in rats, results from rats and other mammalian species suggest that static symptoms subside within $3 \mathrm{~d}$ of the lesion, and this is associated with the recovery of vestibular nuclei resting activity (Ris and Godaux, 1998). Longer-term improvement develops over several weeks, depending on which particular vestibulo-ocular or vestibulo-spinal reflex symptoms are measured (Deliagina et al., 1997). However, some symptoms never compensate completely, even after years of recovery (Gilchrist et al., 1998). To minimize indirect effects associated with the early static symptoms and to examine the long-term effects of permanent vestibular lesions, recordings in the current study were not 
made until at least $60 \mathrm{~d}$ after lesion. At this time-point the symptoms have reached a steady state (Smith and Curthoys, 1989).

Some of the present results have been published previously in abstract form (Russell et al., 2000)

\section{Materials and Methods}

Peripheral vestibular lesion surgery. Twelve naive male Sprague Dawley rats, weighing $\sim 350 \mathrm{gm}$ at the time of surgery, were given either a bilateral labyrinthectomy $(n=6)$ or a sham surgery $(n=6)$. Bilateral labyrinthectomies were conducted under a general anesthetic of sodium pentobarbital $(60 \mathrm{mg} / \mathrm{kg}$, i.p.) by an ear, nose, and throat surgeon (A.H.). The wound margin was anesthetized locally with procaine. Each tympanic bulla was opened by a retro-auricular surgical approach. After removal of the tympanic membrane, malleus, and incus, the vestibule just above the ampullae of the horizontal and anterior semicircular canals was opened with a needle. After aspiration of labyrinthine fluids and the membranous labyrinth from the opened vestibule and the ventral portion of the oval window, the labyrinth was rinsed with $0.1 \mathrm{ml}$ of absolute ethanol perfused through the ventral portion of the oval window and the opened vestibule. For further details, see Zheng et al. (2001). This procedure has been shown to result in complete destruction of the vestibular receptors, as confirmed by temporal bone histology (Fukushima et al., 2001). During labyrinthectomy the auditory system is also destroyed because of the proximity of the vestibular and auditory hair cells. Control animals received a cut to the retro-auricular skin, but the tympanic membrane, malleus, and incus were preserved in the sham operation. All other procedures such as anesthesia and recovery were as for the lesioned animals.

Behavioral observations after labyrinthectomy. All rats were tested behaviorally for vestibular function by assessing the air-righting reflex and contact righting. To test the air-righting reflex, animals were held supine and dropped $\sim 40 \mathrm{~cm}$ onto a soft surface. During the contact-righting test, rats were placed supine on a horizontal surface, and a clear Plexiglas sheet was placed horizontally in contact with their feet. Under both of these circumstances, animals with intact vestibular apparatus will immediately right themselves, whereas animals with bilateral labyrinthectomies will not (Basile et al., 1999).

Electrode implantation surgery. After recovery from the lesion and sham operations (at least 3 weeks), all 12 animals were implanted with a moveable recording electrode that consisted of a bundle of eight $25 \mu \mathrm{m}$ Formvar-coated Nichrome wires. The electrode design, the "scribe microdrive," is described in Bilkey and Muir (1999) and Bilkey et al. (2003). Animals were anesthetized with sodium pentobarbital $(60 \mathrm{mg} / \mathrm{kg}$, i.p.) and mounted in a stereotaxic frame. The electrode was then implanted at $3.8 \mathrm{~mm}$ posterior and $2.5 \mathrm{~mm}$ lateral to bregma (left hemisphere) and 1.8 $\mathrm{mm}$ ventral to the brain surface $(0.2 \mathrm{~mm}$ dorsal to the CAl cell layer) (Paxinos and Watson, 1998). A ground wire was soldered to a stainless steel screw, which was then attached to the skull. The area between the microdrive and the skull was sealed with Vaseline, and the microdrive was cemented in place with dental acrylic, anchored by several skull screws.

Behavioral procedures. Food and water were available ad libitum before and after surgery until the animals had recovered. All rats were then food deprived to reduce their body weight to $85 \%$ of their free-feeding weight and were maintained at this weight throughout the remainder of the study. Recording started a minimum of $60 \mathrm{~d}$ after labyrinthectomy $(70 \pm$ $3.0 \mathrm{~d}$ with no time difference between groups; $t_{10}=0.79 ; p=0.23$ ).

Apparatus. The recording environment consisted of a featureless, smooth, black plastic cylindrical tub, $75 \mathrm{~cm}$ diameter and $56 \mathrm{~cm}$ high. A single, high-intensity, white-shrouded light-emitting diode (LED) observable only from directly below was attached to the top of the wall of the tub and illuminated a spot on the floor that was $\sim 10 \mathrm{~cm}$ in diameter The apparatus was designed in this way to provide a single dominant visual cue, with no olfactory or tactile components, that could be turned off (rather than removed). The tub was situated on a table at a height of 75 $\mathrm{cm}$ and was surrounded by heavy black curtains $(2 \times 2 \mathrm{~m})$ with black PVC plastic on the ceiling, which blocked all light from the windowless recording room. The LED and a lamp on the floor under the table were

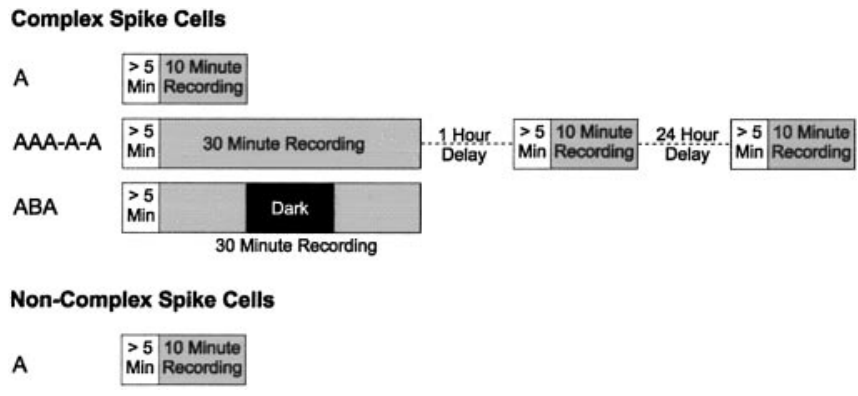

Figure 1. The recording protocols. All recordings consisted of blocks of 10 min sessions. CS cells were recorded for a single session (A), for five sessions with delays (AAA-A-A), or in a light- dark-light (ABA) protocol. Non-CS cells were only recorded for a single session. At least 5 min of habituation time was allowed to elapse before any recording was started.

the only sources of light in the recording environment. Although the rats demonstrated their awareness of the LED by occasionally rearing to investigate, they never demonstrated any aversion to the illuminated area. Sounds were muffled by the curtains and masked by a speaker, tuned to a local radio station, on the center of the ceiling. These measures were taken to ensure that the control rats could not gain an advantage over the deaf lesioned animals by using any auditory cues. Also on the ceiling was an infrared-sensitive video camera, which allowed the position of the animal to be monitored via an infrared LED attached to the headstage of the animal.

Procedure. During a recording session, the animal was carried in a covered opaque box into the recording environment, connected to the recording cable, which was attached to a commutator on the ceiling, and placed in the recording chamber. Fine chocolate chips randomly scattered over the floor of the chamber, and the animal was left to forage freely. Each of the eight electrode wires was checked for unit activity. If no cell was found, then the animal was removed, the microdrive was lowered by $20-40 \mu \mathrm{m}$, and the animal was returned to its home cage for a minimum of $4 \mathrm{hr}$ before retesting.

When a cell was observed it was classified as either a complex spiking (CS) or a noncomplex spiking (non-CS) neuron using criteria that will be described in more detail below. After a 5 min habituation procedure during which the animal was left to forage freely, one of three recording protocols was run (Fig. 1). The first was simply a 10 min session after which the animal was returned to its home cage (A). The second involved three consecutive $10 \mathrm{~min}$ sessions (with no interruption between sessions) followed by a $1 \mathrm{hr}$ delay and another $10 \mathrm{~min}$ session followed by a $24 \mathrm{hr}$ delay and a final $10 \mathrm{~min}$ session (AAA-A-A). The third protocol consisted of three consecutive $10 \mathrm{~min}$ sessions (with no interruption), but during the middle session the room light and LED cue were switched off (ABA). Each animal had experience with all three protocols in a pseudorandom, counterbalanced order. Non-CS cells were recorded only for the first of the three protocols. All CS cells were recorded for the first protocol (A), and a subset of the CS cells was recorded again in the AAA-A-A protocol, the ABA protocol, or both. The decision to record in the latter two protocols was made on the basis of the signal quality and stability. Two CS cells (one control and one lesion) were also recorded for two further sessions, conducted after a 3 week delay and again after 6 weeks (AAA-A-A-A-A) to compare long-term field stability. All details of the protocol were performed in a consistent manner across animals and sessions.

Data acquisition. The electrical signals from the electrodes were buffered with a unity gain field-effect transistor amplifier at the headstage and then amplified, with a gain of 10,000 and filtered, with two-pole active Butterworth high- and low-pass filters, at 300 and $5000 \mathrm{~Hz}$, respectively, through a Barc Neuro8 amplifier. The signal was then sampled in real time using an Axon Instruments DigiData 1200 series digitizer. Putative neuronal spikes were captured by triggering the digitizer every time the signal amplitude exceeded a user-set threshold. When triggered, a 1.5 msec $20 \mathrm{kHz}$ train of 12-bit digitized samples (an event) was obtained starting $0.5 \mathrm{msec}$ before the trigger time. All amplifier, filter, trigger, and 
sampling settings were held constant for a given cell across all sessions within a protocol. The output of the infrared camera was digitized, and a dedicated PC calculated the animal's position. It then generated analog voltages corresponding to the $x$ and $y$ coordinates of the animal. These coordinates were sampled continuously by the digitizer at $60 \mathrm{~Hz}$.

Data analysis. Single neuron spikes were separated from artifacts and noise by setting templates within SpikeX, a purpose-written software program. The data output from SpikeX consisted of time, $x$-position, and $y$-position, captured every $1 / 60$ of a second, and a vector of times at which the neuron spiked. All times were recorded to a resolution of $1 \mathrm{msec}$. Cells were classified electrophysiologically as either CS or non-CS on the basis of the raw waveforms, the interspike interval histogram (histogram of times between adjacent pairs of spikes), and spike time autocorrelation (histogram of times between every pair of spikes). CS cells were those with a spike width of $>400 \mu \mathrm{sec}$ and a spike autocorrelation with a clear peak at 3-8 msec, indicative of complex burst firing. When categorized on the basis of spike width and CS firing, there was a clear bimodal distribution that separated CS from non-CS cells. Cells were included in the analysis only if there were at least 100 spikes during the first recording session corresponding to a minimum of $0.17 \mathrm{~Hz}$. CS cells most likely correspond anatomically to pyramidal cells (Fox and Ranck, 1981; Berger et al., 1983) and functionally to place cells (O'Keefe, 1979). Non-CS cells are most likely a heterogeneous group of GABAergic inhibitory interneurons (Fox and Ranck, 1981; Freund and Buzsaki, 1996). These latter cells are also often referred to as theta cells.

Firing field maps were determined by first dividing the floor of the apparatus into a square $20 \times 20$ grid of pixels, counting the number of spikes at each location $\left(s_{\mathrm{i}}\right)$ and then dividing by the amount of time at that location $\left(t_{\mathrm{i}}\right)$. This resulted in an average firing rate for each pixel $\left(r_{\mathrm{i}}\right)$. Pixels in which the animal spent $<20 \%$ of the average time per pixel were treated as missing data. These parameter choices optimized the compromise between firing rate reliability and the number of excluded pixels. Several properties were then calculated from the resulting average firing rate map, including peak firing rate, mean firing rate, field position, field size, infield/outfield firing ratio, spatial information content (Skaggs et al., 1993), and spatial coherence (Kubie et al., 1990). Each of the firing field measures for the control and lesioned animals was compared using $t$ tests. Note that none of these measures required any special treatment for cells with multiple fields or recordings that may have contained multiple cells with indiscriminable waveforms.

The peak firing rate $\left[\max \left(r_{\mathrm{i}}\right)\right]$ and the mean firing rate $\left(1 / N \Sigma_{\mathrm{i}} r_{\mathrm{i}}\right)$ were measured to characterize the amplitude of the firing field. The field size was defined as the proportion of included pixels with a rate of $\geq 50 \%$ of the peak rate $\left[1 / N\right.$ count $\left.\left(r_{\mathrm{i}} \geq 0.5 r_{\max }\right)\right]$ and the infield/outfield firing ratio as the mean pixel firing rate for all pixels within this subfield divided by the mean pixel firing rate for all other pixels $\left[\left(1 / N_{1} \Sigma_{j} r_{\mathrm{j}}\right) /\left(1 / N_{2} \Sigma_{\mathrm{k}} r_{\mathrm{k}}\right)\right.$, where $r_{\mathrm{j}} \geq 0.5 r_{\max }$ for all $\mathrm{j}$ and $r_{\mathrm{k}}<0.5 r_{\max }$ for all $\mathrm{k}$ and $\left.N_{1}+N_{2}=N\right]$.

To provide measures of spatial firing that do not require the definition of place fields, the data were analyzed using an information content measure (Skaggs et al., 1993) and a spatial coherence measure (Kubie et al., 1990). The information content measure is a quantitative measure of the amount of information (in bits) about location provided by each spike that a cell generates, whereas the spatial coherence measure determines how well clustered in space the pixels of high-firing rate are. These measures indicate the certainty of the animal's position in space as signaled by the cell. Cells with larger fields, lower infield/outfield ratios, lower information contents, or lower spatial coherence convey less information when they fire about the location of the animal.

Finally, the weighted first-order moment of the firing rate map was calculated to determine its center $\left[(X, Y)=1 / N \Sigma_{\mathrm{i}} r_{\mathrm{i}}\left(x_{\mathrm{i}}, y_{\mathrm{i}}\right)\right]$. This was then used to determine whether any shift in field position had occurred. It should be noted that the distributions of possible field location and of possible field shift using this measure, within a bounded region, are far from uniform. However, these distributions are the same for both groups of animals so that direct comparisons can be made.

The stability of the firing fields over time was assessed by calculating the pixel-by-pixel cross-correlation $\left(1 / N\left[\Sigma_{\mathrm{i}}\left[r_{\mathrm{i}}\left(t_{0}\right)-\overline{\mathbf{r}}\left(t_{0}\right)\right]\left[r_{\mathrm{i}}\left(t_{\mathrm{n}}\right)-\right.\right.\right.$ $\left.\left.\left.\overline{\mathbf{r}}\left(t_{\mathrm{n}}\right)\right]\right] /\left[\sigma\left(t_{0}\right) \sigma\left(t_{\mathrm{n}}\right)\right]\right)$ between the firing rate map of the first recording session of each cell with each subsequent session of that cell (AAA-A-A protocol). Exponential regression lines were fitted to the time-varying cross-correlation coefficients, and a two-factor repeated measures ANOVA was also performed on these data.

To analyze the effects of removing visual input (ABA protocol), these data were compared with the first three sessions from the temporal stability experiment (AAA-A-A protocol).

For each measure $\left(m_{\mathrm{k}}\right)$ of the field of each cell, the difference between that measure during the first recording session and each subsequent session was determined $\left[m_{\mathrm{k}}\left(t_{\mathrm{n}}\right)-m_{\mathrm{k}}\left(t_{0}\right)\right]$. The change in each measure as a function of time, its dependence on the lesion, and its dependence on the stability of the environment (AAA or ABA) was then assessed with a three-factor (group, stability-of-environment, and delay) repeated measures ANOVA. The cross-correlations were also analyzed with a threefactor repeated measures ANOVA.

Histological methods. After all recording was completed each animal was deeply anesthetized and a direct current, at $20 \mathrm{~V}$, was passed through the electrodes for $10 \mathrm{sec}$ to mark their final position. The animal was then perfused through the heart with saline $(0.9 \% \mathrm{w} / \mathrm{v})$ followed by a $10 \%$ formalin solution in $0.9 \%$ saline. The brains were removed and stored in formalin solution for a minimum of $2 \mathrm{~d}$ and then transferred to a sucrose formalin solution $(30 \% \mathrm{w} / \mathrm{v})$ for longer-term storage. The brains were sliced into $60 \mu \mathrm{m}$ coronal sections, mounted on glass slides, and stained with thionin. The position of the electrodes was determined by visual inspection under a microscope.

\section{Results}

\section{Behavioral observations}

The behavioral tests confirmed that there was vestibular dysfunction in every lesioned animal but in none of the control animals. In both the air-righting and the contact-righting test, all vestibular lesioned rats failed to right themselves. In contrast, all control animals righted immediately. All lesioned animals also curled into a ball when picked up by the tail and were hyperkinetic, exhibiting increased locomotor activity, typically running repeatedly in circles and swaying their raised head from side to side. These behavioral symptoms decreased in severity or disappeared before the onset of recording. However, the increased locomotor activity persisted throughout the study. The hyperactivity of the lesioned animals is illustrated in Figure $2 A$, which shows the mean speed-distribution $\left[v_{\mathrm{i}}=1 / \Delta t \sqrt{ }\left(\left(x_{\mathrm{i}+1}-x_{\mathrm{i}}\right)+\left(y_{\mathrm{i}+1}-\right.\right.\right.$ $\left.\left.\left.y_{\mathrm{i}}\right)\right)\right]$ over all sessions. Observation of the animals indicated, however, that the lesioned animals' exploratory behavior was otherwise relatively normal. This was confirmed by a comparison between groups of the proportion of the environment visited during each session $\left(N / N_{\text {total }}\right)$ (Fig. $2 B$ ). In addition, there was no difference in the heterogeneity of exploration, as assessed by the coefficient of variation of pixel dwell times $\left[\sigma\left(t_{\mathrm{i}}\right) / \operatorname{mean}\left(t_{\mathrm{i}}\right)\right]$ (Fig. $2 C$ ).

\section{Histology and cell numbers}

The results of the histology showed that all electrodes had passed through the CA1 cell layer of the dorsal hippocampus. A total of 113 cells were recorded, 80 of which were classified as CS neurons. Control and lesion groups had approximately equal cell numbers for each cell type, with no significant difference revealed by a $\chi^{2}$ analysis. The distributions of cells per animal were reasonably broad but were similar between the two groups. The range was 3-11 cells for control animals and 2-11 cells for lesioned animals, with a coefficient of variation of 0.44 and 0.50 , respectively. The number and type of cells recorded for control and lesion animals in each of the three protocols are given in Table 1.

\section{Firing characteristics of hippocampal neurons}

The overall mean firing rate $\left(\Sigma_{\mathrm{i}} s_{\mathrm{i}} / \Sigma_{\mathrm{i}} t_{\mathrm{i}}\right)$ of hippocampal cells was higher in the lesioned animals as compared with controls for both 


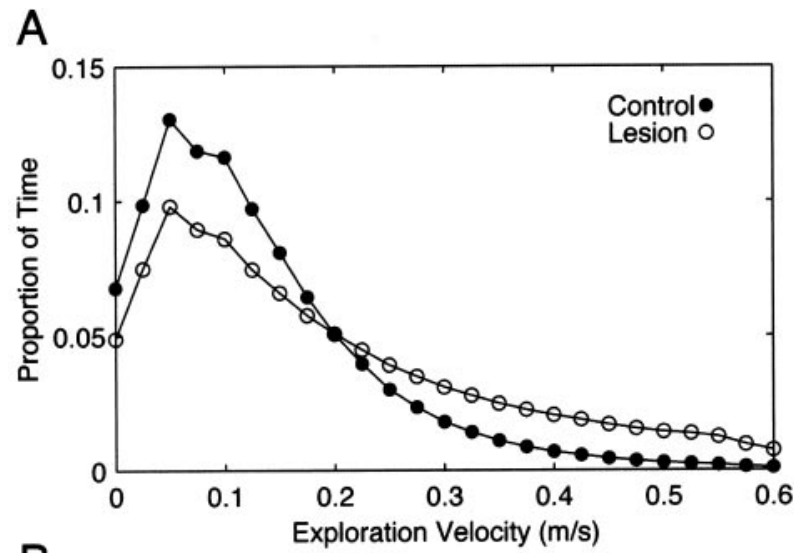

B

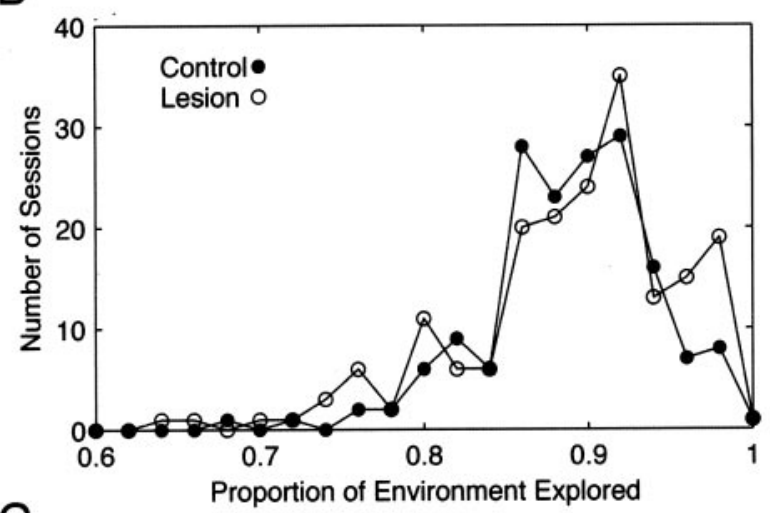

$\mathrm{C}$

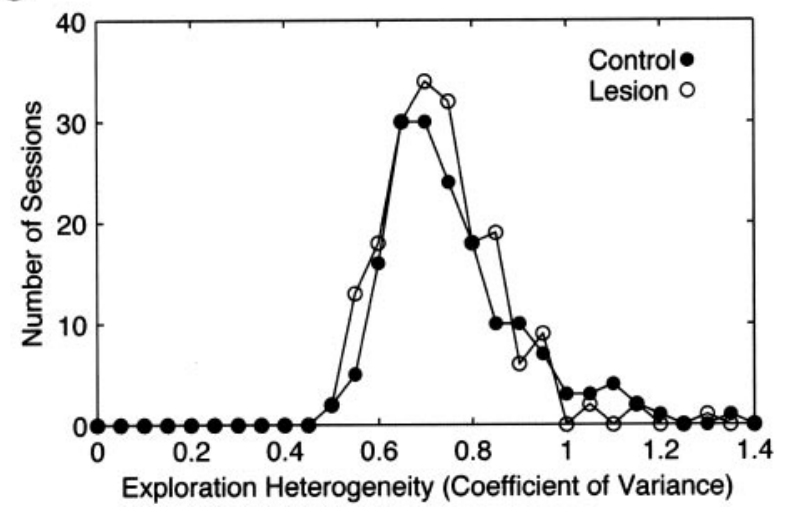

Figure 2. Comparison of the behavior of control and lesioned animals. A, Mean velocity distributions over all control sessions $(n=171)$ and all lesion sessions $(n=193)$. Note that the lesioned animals spend a greater proportion of the time moving faster, reflecting their hyperactivity. The SE bars have been omitted from this figure because the values were so low that they interfered with the clarity of the symbols denoting the two groups. Despite their hyperactivity, the lesioned animals did not differ in their coverage of the environment during exploration $(B)$ or in the heterogeneity of their exploration as assessed by the coefficient of variation of pixel dwell times $(C)$.

CS cells (control, $2.42 \pm 0.35 \mathrm{~Hz}$; lesion, $3.91 \pm 0.65 \mathrm{~Hz} ; t_{78}=$ $2.04 ; p=0.022$ ) and non-CS cells (control, $14.9 \pm 2.1 \mathrm{~Hz}$; lesion, $\left.22.0 \pm 3.2 \mathrm{~Hz} ; t_{31}=1.82 ; p=0.039\right)$. Complex spike bursts, and the tendency for the cell to fire approximately every $120 \mathrm{msec}$, appeared to be similar in both the control and the lesion group as determined by examination of the raw waveform, the mean histogram of interspike intervals, and the mean autocorrelation functions. It is unlikely that the hyperactivity of the lesioned animals caused any electrode drift or destabilized cells because the spike waveforms were stable both within and between recording sessions of the same cell in both groups of animals, and there was
Table 1. The number of CS and non-CS cells recorded from each group of animals in each protocol

\begin{tabular}{lll}
\hline & $\begin{array}{l}\text { Number of control cells } \\
\text { (6 animals) }\end{array}$ & $\begin{array}{l}\text { Number of lesion cells } \\
\text { (6 animals) }\end{array}$ \\
\hline CS cells & & \\
A & 41 & 39 \\
AAA-A-A & 19 & 22 \\
ABA & 14 & 11 \\
Non-CS cells & & 22 \\
A & 11 & \\
\hline
\end{tabular}

Table 2. Firing field properties of both CS and non-CS cells for each group of animals

\begin{tabular}{lcc}
\hline & Control & Lesion \\
\hline CS cells & & \\
$\quad$ Peak firing rate (Hz) & $17.7 \pm 1.5$ & $16.8 \pm 1.4$ \\
Mean firing rate (Hz) & $2.36 \pm 0.32$ & $4.05 \pm 0.69^{*}$ \\
Field size (\%) & $5.0 \pm 0.5$ & $9.5 \pm 1.8^{*}$ \\
Infield/outfield firing ratio & $11.7 \pm 2.3$ & $5.32 \pm 0.51^{* *}$ \\
Information content & $1.18 \pm 0.11$ & $0.57 \pm 0.07^{* * *}$ \\
$\quad$ Spatial coherence & $0.57 \pm 0.03$ & $0.35 \pm 0.03^{* * *}$ \\
Non-CS cells & & \\
Peak firing rate (Hz) & $34.5 \pm 4.5$ & $42.8 \pm 5.9$ \\
Mean firing rate (Hz) & $15.3 \pm 2.3$ & $22.5 \pm 3.3^{*}$ \\
Field size (\%) & $36.1 \pm 6.7$ & $51.2 \pm 5.1^{*}$ \\
Infield/outfield firing ratio & $2.07 \pm 0.42$ & $1.66 \pm 0.09$ \\
Information content & $0.094 \pm 0.023$ & $0.069 \pm 0.019$ \\
Spatial coherence & $0.343 \pm 0.051$ & $0.269 \pm 0.027$ \\
\hline
\end{tabular}

Mean \pm SEM is listed with significant differences between control and lesion animals indicated by ${ }^{*} p<0.05$ ${ }^{* *} p<0.01$, or ${ }^{* * *} p<0.001$.

no apparent difference in signal-to-noise of the raw waveform between the two groups. Furthermore, this electrode-microdrive assembly has been shown previously to provide stable data in the mechanically more stressful situation in which pigeons were continuously pecking response keys during the unit recording procedure (Bilkey et al., 2003).

\section{Spatial firing correlations of hippocampal neurons}

CS cell firing fields were significantly larger in lesioned animals with significantly lower infield/outfield firing ratios, significantly higher mean firing rates, but no difference in peak firing rates (Table 2). An analysis of information content showed that lesion CS cells provided substantially fewer bits of spatial information per spike compared with controls, and the spatial coherence was also significantly lower for cells from lesioned animals than for controls (Table 2).

In addition, the mean firing rate, but not the peak firing rate, was higher and the field size was larger for non-CS cells (Table 2). Note that the mean pixel firing rate and the overall mean firing rate will be equal only if the animal spends the same amount of time in every pixel, and therefore the difference in these measures provides an index of the heterogeneity in the animal's exploration. Qualitative examination of the firing fields confirmed that lesion fields are larger and less uniform than control fields. Figure 3 shows representative examples. Also included for comparison are control and lesion non-CS cell fields (Fig. 4). These examples were selected objectively by finding the field with the minimum sum of squares of the difference from the mean over all six field measures (Table 2) for control animals' firing fields $\left(\min _{\mathrm{n}}\left[\Sigma_{\mathrm{k}}\right.\right.$ $\left.\left(m_{\mathrm{kn}}-\overline{\mathbf{m}}_{\mathbf{k}}\right)^{2}\right]$, where $\overline{\mathbf{m}}_{\mathbf{k}}$ is the mean of measure $m_{\mathrm{k}}$ over $n$ control animal fields) and for lesioned animal firing fields $\left(\min _{\mathrm{n}}\left[\Sigma_{\mathrm{k}}\right.\right.$ 


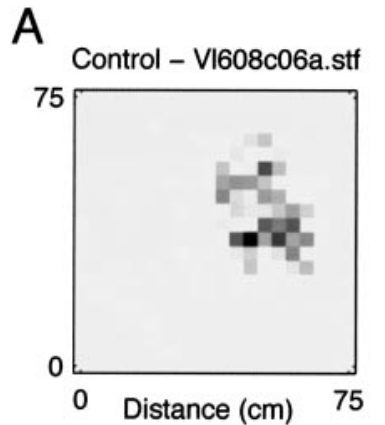

B
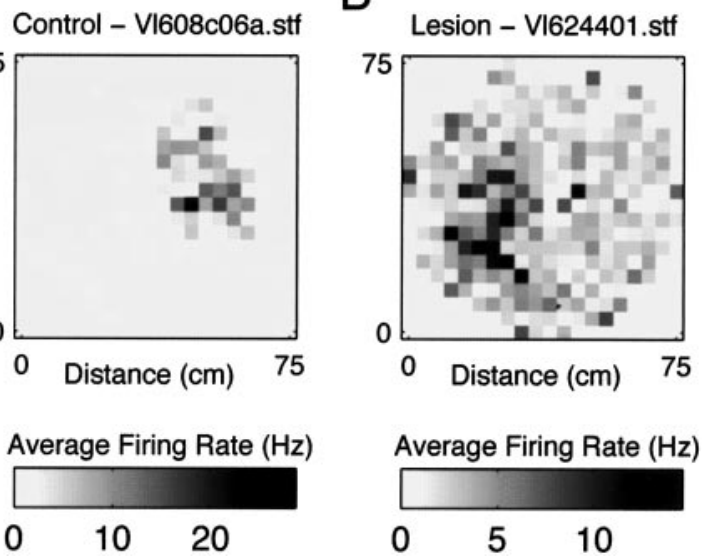

Figure 3. Representative firing rate maps for CS cells in control $(A)$ and lesion $(B)$ animals. Note that the spatially dependent firing for the lesioned animals is less well defined. The fields shown were selected objectively by finding the field with properties that were closest to average.
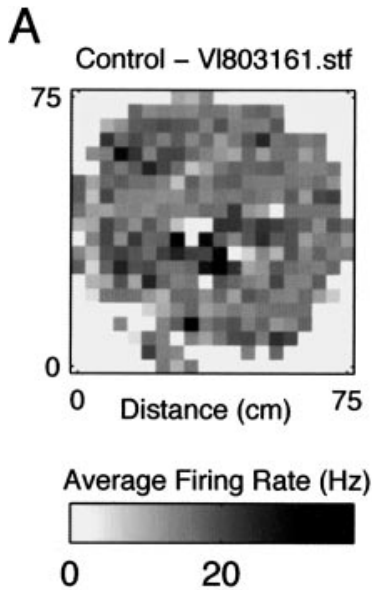

B
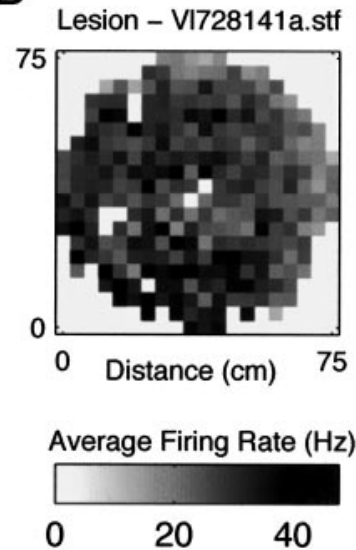

Figure 4. Representative firing rate maps for non-CS cells in control $(A)$ and lesion $(B)$ animals. Although the spatial firing correlates are very weak for non-CS fields, they do exist. The fields shown were selected objectively by finding the field with properties that were the closest to average.

$\left.\left(m_{\mathrm{kn}}-\overline{\mathbf{m}}_{\mathbf{k}}\right)^{2}\right]$ where $\overline{\mathbf{m}}_{\mathbf{k}}$ is the mean of measure $m_{\mathrm{k}}$ over $n$ lesioned animal fields).

\section{Stability of spatial firing correlations with time}

There was a very clear difference between control and lesion animals' field cross-correlations (Fig. $5 A$ ) where there were significant group $\left(F_{(1,39)}=54.5 ; p<0.0001\right)$, delay $\left(F_{(3,117)}=10.7 ; p<\right.$ $0.0001)$, and interaction $\left(F_{(3,117)}=3.1 ; p=0.031\right)$ effects. Both lesion and control cross-correlation coefficients were described by an exponential decay function (control, $r=0.42, p=0.0002$; lesion, $r=0.26, p=0.016$ ) with no significant difference between the decay constants. The steady decline in cross-correlation with time continued over a period of 6 weeks for the two cells recorded at long delays (Fig. 5B). An exponential decay function also described the decay in cross-correlation coefficients of these two cells (control, $r=0.89, p=0.017$; lesion, $r=0.92, p=0.0097$ ). The control and lesion animal firing fields recorded over this 6 week period are illustrated in Figure 6.

On the basis of the field properties and cross-correlation analysis, it appears that there may be some spatially dependent firing
A

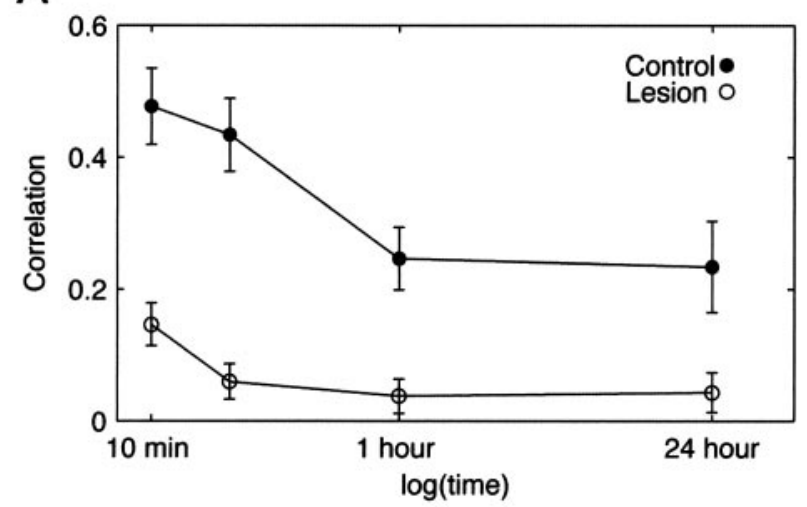

B

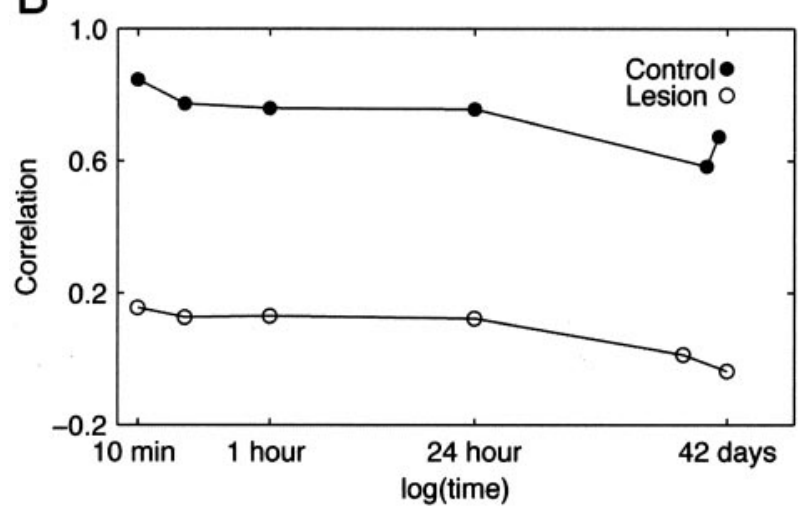

Figure 5. Firing rate map cross-correlations between sessions in the AAA-A-A protocol. Lesion maps had lower cross-correlations, indicating instability over a period of several minutes. $A$, Mean and SEM cross-correlations over all cells. $B$, Cross-correlations from one control and one lesion cell recorded for 6 weeks.

activity present in the lesioned animals. To investigate this possibility more carefully, further analysis was performed. Because the lesion group's cross-correlation decreases almost immediately (within $10 \mathrm{~min}$ ), further analysis was run on the $30 \mathrm{~min}$ duration, continuous recording sessions (AAA) to improve the temporal resolution of this analysis. These sessions were split into six $5 \mathrm{~min}$ sessions instead of three 10 min sessions. Cross-correlation analyses performed on the six 5 min sessions shows that the correlations are near noise level even when the first two 5 min sessions are compared (Fig. 7A). Cross-correlation analysis on ten $3 \mathrm{~min}$ sessions yielded the same results, although the amount of missing data in this analysis make the results less reliable. Cumulative firing fields were then calculated using data from the start of the recording session $\left(t_{0}\right)$ to a later time $t_{\mathrm{n}}$, where $t_{\mathrm{n}}$ varied from 5 to $30 \mathrm{~min}$ in $5 \mathrm{~min}$ steps. Figure $7 B$ shows a plot of the cumulative firing field size as the $30 \mathrm{~min}$ session progresses. The control animals' fields are well established within the first $5 \mathrm{~min}$ and do not increase in size with time. In contrast, the lesioned animal's cumulative field continues to increase in size during the $30 \mathrm{~min}$ recording without clearly reaching an asymptote. Collectively these results suggest that the firing activity of the lesion-group cells is not locked to the animal's location in space, and although the presence of some residual spatially dependent firing cannot be ruled out, it is likely that spatially dependent firing in animals with bilateral labyrinthectomies is completely abolished.

Stability of spatial firing correlations in the dark

Turning off the lights did not have an effect on the stability of firing fields of either lesion or control animals. Thus although 
A
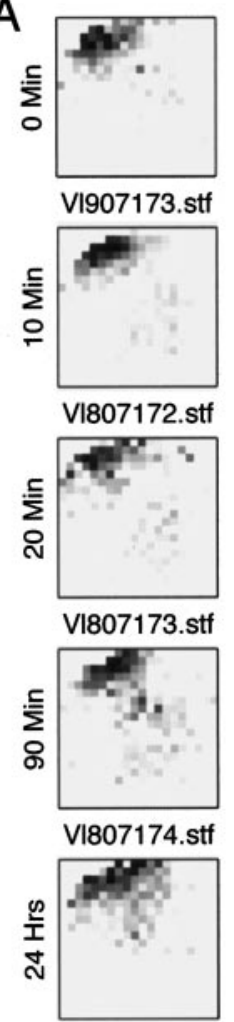

VI807175.stf

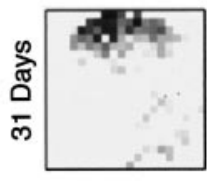

VI907171.stf

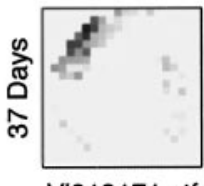

VI913171.stf
B

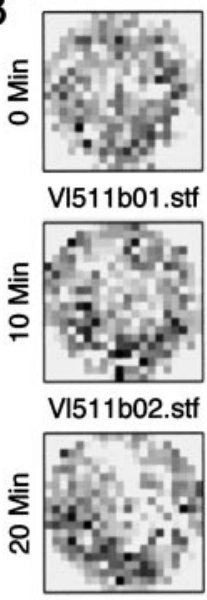

VI511b03.stf

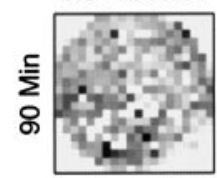

VI511b04.stf

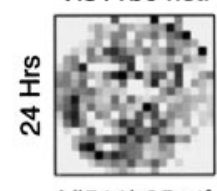

V1511b05.stf

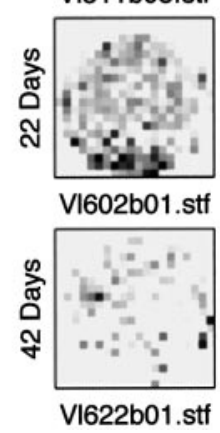

Figure 6. Firing rate maps for the cells recorded over a 6 week period shown in Figure 5B.A, Control CS cell. B, Lesion CS cell.

both lesion and control animals' fields shifted in the dark, this was comparable with the corresponding shifts seen in the light (Fig. 8 ). The analysis of variance on the field measures showed that there was very little delay effect and that this was not group dependent.

Of the five ANOVAs performed, only 7 of 35 results achieved $5 \%$ significance, and this reduced to 3 significant results after making a Bonferroni multiple measures correction. Of the results that reached 5\% significance, there was a group difference in the change to infield/outfield firing ratio, with lesion fields having lower values $\left(F_{(1,78)}=8.58 ; p=0.004\right)$. Delay effects were seen for the change to mean rate $\left(F_{(1,78)}=9.61 ; p=0.003\right)$, the change to infield/outfield firing ratio $\left(F_{(1,78)}=17.7 ; p<0.0001\right)$, and the change to the shift of the field's position $\left(F_{(1,78)}=5.73 ; p=\right.$ $0.019)$. Most importantly, however, there was no stability-ofenvironment effect, i.e., the change to field measures in the lightdark-light condition was no different from the changes that occurred in the light-light-light condition. This result suggests that visual information was not necessary for the maintenance of an

A

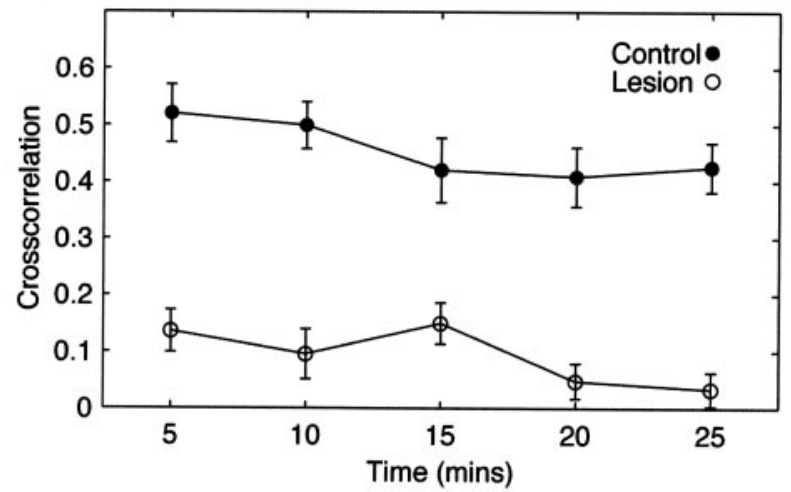

B

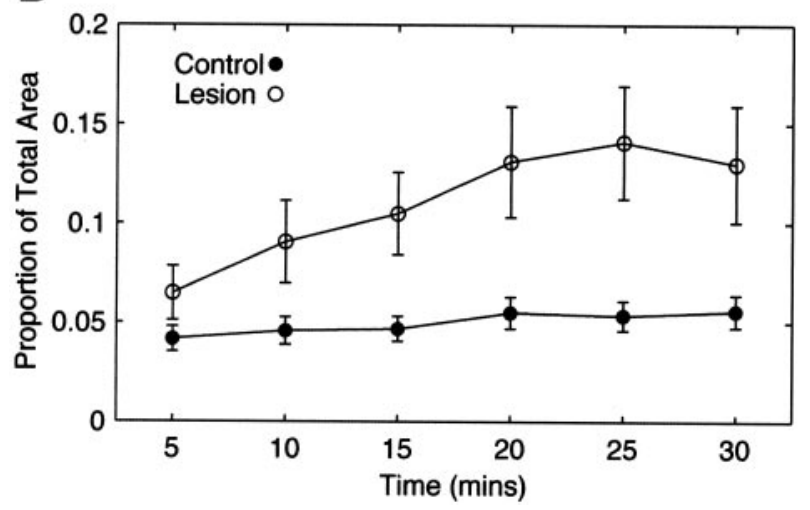

Figure 7. Changes in spatially dependent firing in the lesion group cells over time. $A$, Crosscorrelations between the firing rate map recorded during the first 5 min block of a 30 min continuous recording session and each subsequent 5 min block. Lesion group maps have low cross-correlations immediately on the first comparison, indicating instability over a period of a few minutes. $B$, Mean cumulative place field size. Field size was calculated from firing data between $t_{0}$ and a later time $t_{n}$, where $t_{n}$ increased in 5 min increments from 5 to 30 min. Control group fields reach maximum size within $5 \mathrm{~min}$ with no further cumulative increase. In contrast, lesion group fields continue to increase in size with no obvious asymptote. Collectively these results suggest that there is no spatially dependent firing in the lesioned animals.

accurate representation of space in control animals and yields no extra information for lesioned animals. Group $\times$ delay interactions were seen for changes to the mean rate $\left(F_{(1,78)}=4.06 ; p=\right.$ $0.047)$ and changes to the infield/outfield firing ratio $\left(F_{(1,78)}=\right.$ $4.45 ; p=0.038)$, and finally a stability-of-environment $\times$ delay interaction was seen for the changes to peak rate $\left(F_{(1,78)}=4.98\right.$; $p=0.029)$. To summarize, there was predominantly no change to the stability of firing fields in the absence of vision.

In the cross-correlation analysis there were clear group $\left(F_{(1,78)}\right.$ $=46.9 ; p<0.0001)$ and delay $\left(F_{(1,78)}=6.56 ; p=0.012\right)$ effects, but no effect caused by the change in lighting. Significant group $\times$ stability-of-environment $\left(F_{(1,78)}=9.85 ; p=0.002\right)$, group $\times$ delay $\left(F_{(1,78)}=14.4 ; p=0.0003\right)$, and group $\times$ delay $\times$ stability-of-environment $\left(F_{(1,78)}=7.67 ; p=0.007\right)$ interaction effects were observed.

\section{Discussion}

The results of the present study reveal that a sudden and complete permanent bilateral vestibular labyrinthectomy, made by a surgical-chemical procedure, produces major changes in the firing of both CS and non-CS neurons of the hippocampal CA1 region. In particular, the spatial correlates of CS cell firing appeared to be completely abolished in the lesioned animals. These results are consistent with the hypothesis that the hippocampus uses self- 
A

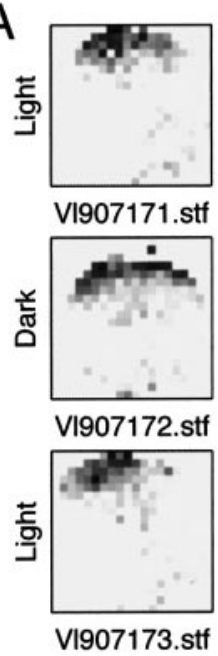

B

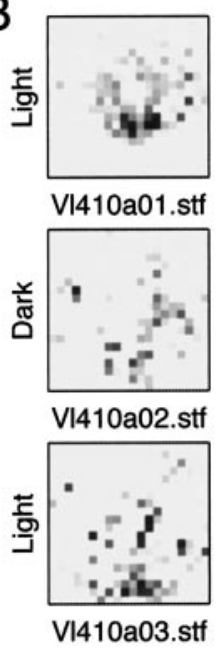

Figure 8. Firing rate maps recorded in the light-dark-light (ABA) protocol in control $(A)$ and lesion $(B)$ animals. The changes to the properties of the firing field that occurred in the dark were no greater than the changes in the light, over the same time period, for either control or lesioned animals. Hence the observed changes to the lesion animal's field in the dark cannot be attributed to the absence of vision.

movement information from the vestibular system to modulate place cell firing.

The results of the present study confirm those of Stackman et al. (2002). The use of transient lesions in their study may have unduly stressed the animal or disrupted attentional mechanisms and indirectly resulted in changes to hippocampal cell activity. The accord between their findings and those of the present study, however, indicates that the previous results were not simply caused by indirect consequences of the lesion. There were some differences between the results of the current study and those of Stackman et al. (2002). For instance, they reported no effect on cell firing rates of theta cells (putative interneurons). It is possible that the increase in cell firing rates observed in the current study reflects changes related to vestibular compensatory processes. For example, the permanent damage to the two vestibular labyrinths would ultimately result in the degeneration of the vestibular nerve fibers within the vestibular nuclei (Smith and Curthoys, 1989), potentially leading to glial cell-induced neurochemical changes that could affect other areas of the CNS. Such changes might not be seen with transient or incomplete lesions.

A key question is whether the disruption to the spatial firing correlates reflects the loss of a primary vestibular contribution to hippocampal function or whether it is a secondary consequence of lesion-induced behavioral or sensory dysfunction. Information flow can be disrupted in a number of ways. First, signals from the affected sensory modality are abolished completely. Second, other sensory modalities may have abnormal distributions of otherwise normal signals as a result of a change in behavior. For example, the animal may adopt an unusual posture, resulting in an increase in unusual but noncorrupted proprioceptive and tactile signals. Changes may also occur to CNS networks to accommodate this shift in sensory distributions. Third, signals from other sensory modalities may be disrupted. Visual field stability during motion is dependent, for example, on having an intact vestibulo-ocular function (for review, see Curthoys and Halmagyi, 1995).

Issues of primary versus secondary effects are of course problematic for any lesion study and will always make interpretation difficult. We can discount, however, many explanations that are

based on secondary effects. With regard to absent inputs, for example, it is possible that the current results are caused by the loss of hearing in the lesioned animals. Hill and Best (1981), however, failed to report a difference in place field properties in deaf rats. Furthermore, Rossier et al. (2000) demonstrated that rats are unable to use auditory cues alone to support spatial navigation.

Bilateral vestibular loss results in a collapse of spontaneous resting activity in the two vestibular nuclei, causing severe postural symptoms such as ataxia. However, as resting activity regenerates in the vestibular nuclei over several days after the lesion (Ris and Godaux, 1998), these early static symptoms subside, and what remains are the long-term deficits in the responses of the vestibular reflexes to head movement (Smith and Curthoys, 1989). The severity of the initial static symptoms in particular could conceivably result in the feedback of abnormal sensory signals to the hippocampus. Although motion and postural disturbances in the lesioned animals are potential confounds that are difficult to eliminate, several pieces of evidence suggest that they may not be having a major effect on cell firing. For example, it has been shown previously that the degree of velocity modulation of CS cell firing is relatively small (McNaughton et al., 1983). Furthermore, the orientation of a rat as it moves through the place field appears to have little effect on the firing in the open field (Markus et al., 1995), and it has been shown that place fields are still apparent in rats that are moved passively through the environment (Gavrilov et al., 1998). Interestingly, lesions of both the vestibular system (Basile et al., 1999) and the septohippocampal system (Bannerman et al., 2001) are known to cause hyperkinesis.

Vestibular signals control the positioning and movement of the eyes, via the vestibular ocular reflex (VOR) and optokinetic reflexes, to stabilize the visual field on the retina during head movements. In the lesioned animals the lack of a VOR will cause the visual field to slip (oscillopsia) during large head accelerations (for review, see Smith and Curthoys, 1989; Curthoys and Halmagyi, 1995). Because visual inputs have been shown to have control over place fields (Shapiro et al., 1997) and visual flow information reaches the hippocampus via the accessory optic system in pigeons and possibly in rats (Wylie et al., 1999), an unstable visual field could potentially disrupt spatial representations by limiting the animals' ability to use visual cues to orient themselves. The current finding that the absence of visual input did not affect the stability of the firing fields indicates, however, that vision on its own is not a prerequisite for spatial firing. This is consistent with previous findings (Quirk et al., 1990; Markus et al., 1994). For example, it has been shown that blind rats can form and maintain place fields as well as sighted rats (Save et al., 1998).

Previous data suggest that when an animal is in the dark, place fields are more reliant on self-motion cues (Gothard et al., 2001). This may include vestibular signals as well as proprioceptive, motor efferent, or sensory flow cues, for example, the flow of olfactory cues as the animal moves or airflow over their vibrissae. On the basis of the present data, however, it appears that vestibularlesioned animals are unable to use these nonvestibular selfmotion cues to localize themselves, indicating that vestibulardependent self-motion signals are critical for the maintenance of hippocampal spatial firing activity.

In the present study the mechanism responsible for complex spike bursts was unaffected by lesions to the vestibular apparatus, and the temporal distribution of firing appeared normal. Therefore, in the lesioned animals, the mechanisms that result in sporadic bursts of activity from CS neurons are intact, whereas the 


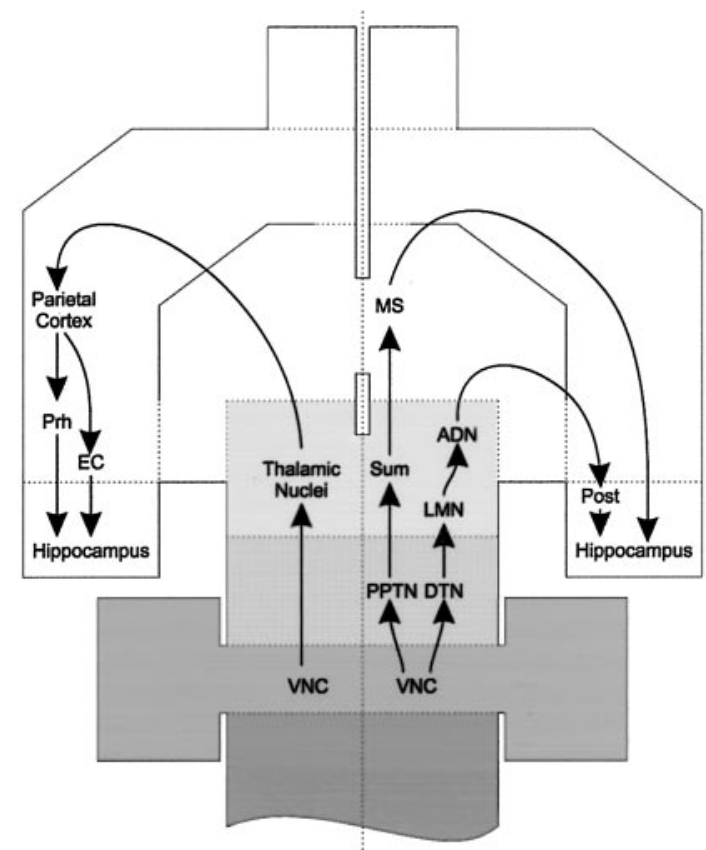

Figure 9. Potential polysynaptic anatomical pathways from the VNC to the hippocampus. The thalamocortical route is shown on the left, and the septohippocampal and head direction system routes are shown on the right for clarity only. The pathways have been drawn onto a scale two-dimensional topographical projection of the rat brain. The different shaded regions indicate, from bottom to top, the myencephalon, metencephalon, mesencephalon, diencephalon, and telencephalon, respectively. VNC, Vestibular nucleus complex; Prh, perirhinal cortex; EC, entorhinal cortex; PPTN, pendunculopontine tegmental nucleus; Sum, supramammillary nucleus; MS, medial septum; DTN, dorsal tegmental nucleus; LMN, lateral mammillary nucleus; AND, anterodorsal thalamic nucleus; Post, postsubiculum.

mechanisms that constrain this activity to a specific location in space are not. Because the temporal distribution of firing was intact in the lesioned animals, a nonuniform distribution of spatial firing resulted when activity was integrated over a sufficiently short time interval (i.e., $10 \mathrm{~min}$ ). Hence the firing fields in the lesioned animals had a higher variance than expected from temporally uniform random firing. This resulted in smaller field sizes, higher infield/outfield ratios, and higher information content than expected from random firing. This signal could be used in a spatially meaningful way, however, only if it is stable over time. In the lesioned animals the spatial dependence of firing activity was not stable, even over time periods of a few minutes. We conclude, therefore, that it is likely that the spatial signal encoded in the firing of hippocampal cells has been abolished completely.

In summary, the results of the current experiment, when taken in conjunction with the findings of the previous transient lesion study and those of studies that have indirectly manipulated vestibular input to the hippocampus, provide converging evidence demonstrating that vestibular information is processed by the hippocampus. Vestibular information could potentially be routed to the hippocampus through one or more different polysynaptic pathways (Fig. 9). It may ascend via a thalamocortical route (Smith, 1997), through theta rhythm-generating structures leading to the medial septum (Semba et al., 1988; Kirk and McNaughton, 1991; Hayakawa et al., 1993; Vertes and Kocsis, 1997), or via the "head direction" system (Taube et al., 1996). For example, spatially dependent firing may have been corrupted in the lesioned animals because of disruption to the head direction system, because it has been shown that sodium arsanilate- induced vestibular system lesions abolish head direction cell firing in the anterior thalamic nucleus (Stackman and Taube, 1997). Other possible polysynaptic pathways exist by which a disruption of the vestibular input to the hippocampus might interrupt the reconciliation of internal self-movement signals with the changes to the external sensory inputs that occur as a result of that movement. This would disrupt the ability of the animal to integrate allocentric and egocentric information into a coherent representation of space. Because it is known that the hippocampus is also involved in nonspatial functions (Wood et al., 2001), the effect that vestibular signals have on this activity would also be of considerable interest.

\section{References}

Bannerman DM, Gilmour G, Norman G, Lemaire M, Iversen SD, Rawlins JN (2001) The time course of the hyperactivity that follows lesions or temporary inactivation of the fimbria-fornix. Behav Brain Res 120:1-11.

Basile AS, Brichta AM, Harris BD, Morse D, Coling D, Skolnick P (1999) Dizocilpine attenuates streptomycin-induced vestibulotoxicity in rats. Neurosci Lett 265:71-74.

Berger TW, Rinaldi PC, Weisz DJ, Thompson RF (1983) Single-unit analysis of different hippocampal cell types during classical conditioning of rabbit nictitating membrane response. J Neurophysiol 50:1197-1219.

Bilkey DK, Muir GM (1999) A low cost, high precision subminiature microdrive for extracellular unit recording in behaving animals. J Neurosci Methods 92:87-90.

Bilkey DK, Russell NA, Colombo M (2003) A lightweight microdrive for single unit recording in freely moving rats and pigeons. Methods 30:152-158.

Curthoys IS, Halmagyi GM (1995) Vestibular compensation: a review of the oculomotor, neural, and clinical consequences of unilateral vestibular loss. J Vestib Res 5:67-107.

Cuthbert PC, Gilchrist DP, Hicks SL, MacDougall HG, Curthoys IS (2000) Electrophysiological evidence for vestibular activation of the guinea pig hippocampus. NeuroReport 11:1443-1447.

Deliagina TG, Popova LB, Grant G (1997) The role of tonic vestibular input for postural control in rats. Arch Ital Biol 135:239-261.

Dudchenko P, Goodridge JP, Taube JS (1997) The effects of disorientation on visual landmark control of head direction cell orientation. Exp Brain Res 115:375-380.

Fox SE, Ranck Jr JB (1981) Electrophysiological characteristics of hippocampal complex-spike cells and theta cells. Exp Brain Res 41:399-410.

Freund TF, Buzsaki G (1996) Interneurons of the hippocampus. Hippocampus $6: 347-470$.

Fukushima M, Kitahara T, Takeda N, Saika T, Uno A, Kubo T (2001) Role of cholinergic mossy fibers in medial vestibular and prepositus hypoglossal nuclei in vestibular compensation. Neuroscience 102:159-166.

Gavrilov VV, Wiener SI, Berthoz A (1998) Discharge correlates of hippocampal complex spike neurons in behaving rats passively displaced on a mobile robot. Hippocampus 8:475-490.

Gilchrist DPD, Curthoys IS, Cartwright AD, Burgess AM, Topple AN, Halmagyi GM (1998) High acceleration impulsive rotations reveal severe long-term deficits of the horizontal vestibulo-ocular reflex in the guinea pig. Exp Brain Res 123:242-245.

Gothard KM, Hoffman KL, Battaglia FP, McNaughton BL (2001) Dentate gyrus and CAl ensemble activity during spatial reference frame shifts in the presence and absence of visual input. J Neurosci 21:7284-7292.

Hayakawa T, Ito H, Zyo K (1993) Neuroanatomical study of afferent projections to the supramammillary nucleus of the rat. Anat Embryol 188:139-148.

Hill AJ, Best PJ (1981) Effects of deafness and blindness on the spatial correlates of hippocampal unit activity in the rat. Exp Neurol 74:204-217.

Jensen DW (1983) Survival of function in the deafferented vestibular nerve. Brain Res 273:175-178.

Kirk IJ, McNaughton N (1991) Supramammillary cell firing and hippocampal rhythmical slow activity. NeuroReport 2:723-725.

Kubie JL, Muller RU, Bostock E (1990) Spatial firing properties of hippocampal theta cells. J Neurosci 10:1110-1123.

Markus EJ, Barnes CA, McNaughton BL, Gladden VL, Skaggs WE (1994) Spatial information content and reliability of hippocampal CA1 neurons: effects of visual input. Hippocampus 4:410-421. 
Markus EJ, Qin YL, Leonard B, Skaggs WE, McNaughton BL, Barnes CA (1995) Interactions between location and task affect the spatial and directional firing of hippocampal neurons. J Neurosci 15:7079-7094.

McNaughton BL, Barnes CA, O’Keefe J (1983) The contributions of position, direction, and velocity to single unit activity in the hippocampus of freely-moving rats. Exp Brain Res 52:41-49.

Muller RU, Kubie JL, Ranck Jr JB (1987) Spatial firing patterns of hippocampal complex-spike cells in a fixed environment. J Neurosci 7:1935-1950.

O'Keefe J (1979) A review of the hippocampal place cells. Prog Neurobiol 13:419-439.

O'Keefe J, Dostrovsky J (1971) The hippocampus as a spatial map. Preliminary evidence from unit activity in the freely-moving rat. Brain Res 34:171-175.

O'Keefe J, Nadel L (1978) The hippocampus as a cognitive map. Oxford: Clarendon.

O’Mara SM, Rolls ET, Berthoz A, Kesner RP (1994) Neurons responding to whole-body motion in the primate hippocampus. J Neurosci 14:6511-6523.

Paxinos G, Watson C (1998) The rat brain in stereotaxic coordinates, Ed 4. Orlando, FL: Academic.

Quirk GJ, Muller RU, Kubie JL (1990) The firing of hippocampal place cells in the dark depends on the rat's recent experience. J Neurosci 10:2008-2017.

Ris L, Godaux E (1998) Neuronal activity in the vestibular nuclei after contralateral or bilateral labyrinthectomy in the alert guinea pig. J Neurophysiol 80:2352-2367.

Rossier J, Haeberli C, Schenk F (2000) Auditory cues support place navigation in rats when associated with a visual cue. Behav Brain Res 117:209-214

Russell NA, Horii A, Liu P, Smith PF, Darlington CL, Bilkey DK (2000) Hippocampal place Fields have decreased stability in rats with bilateral vestibular labyrinthectomies. Soc Neurosci Abstr 26:173.4.

Save E, Cressant A, Thinus-Blanc C, Poucet B (1998) Spatial firing of hippocampal place cells in blind rats. J Neurosci 18:1818-1826.

Semba K, Reiner PB, McGeer EG, Fibiger HC (1988) Brainstem afferents to the magnocellular basal forebrain studied by axonal transport, immunohistochemistry, and electrophysiology in the rat. J Comp Neurol 267:433-453.

Shapiro ML, Tanila H, Eichenbaum H (1997) Cues that hippocampal place cells encode: dynamic and hierarchical representation of local and distal stimuli. Hippocampus 7:624-642.

Sharp PE, Blair HT, Etkin D, Tzanetos DB (1995) Influences of vestibular and visual motion information on the spatial firing patterns of hippocampal place cells. J Neurosci 15:173-189.

Skaggs WE, McNaughton BL, Gothard KM, Markus EJ (1993) An informationtheoretic approach to deciphering the hippocampal code. In: Advances in neural information processing systems (Hanson SJ, Cowan JD, Giles CL, eds), pp 1030-1037. San Mateo, CA: Morgan Kaufmann.

Smith PF (1997) Vestibular-hippocampal interactions. Hippocampus 7:465-471.

Smith PF, Curthoys IS (1989) Mechanisms of recovery following unilateral labyrinthectomy: a review. Brain Res Brain Res Rev 14:155-180.

Stackman RW, Taube JS (1997) Firing properties of head direction cells in the rat anterior thalamic nucleus: dependence on vestibular input. J Neurosci 17:4349-4358.

Stackman RW, Clark AS, Taube JS (2002) Hippocampal spatial representations require vestibular input. Hippocampus 12:291-303.

Taube JS, Goodridge JP, Golob EJ, Dudchenko PA, Stackman RW (1996) Processing the head direction cell signal: a review and commentary. Brain Res Bull 40:477-486.

Vertes RP, Kocsis B (1997) Brainstem-diencephalo-septohippocampal systems controlling the theta rhythm of the hippocampus. Neuroscience 81:893-926.

Whishaw IQ (1998) Place learning in hippocampal rats and the path integration hypothesis. Neurosci Biobehav Rev 22:209-220.

Wiener SI, Korshunov VA, Garcia R, Berthoz A (1995) Inertial, substratal and landmark cue control of hippocampal CA1 place cell activity. Eur J Neurosci 7:2206-2219.

Wilson VJ, Melvill Jones G (1979) Mammalian vestibular physiology. New York: Plenum.

Wood ER, Dudchenko PA, Eichenbaum H (2001) Cellular correlates of behavior. Int Rev Neurobiol 45:293-312.

Wylie DR, Glover RG, Aitchison JD (1999) Optic flow input to the hippocampal formation from the accessory optic system. J Neurosci 19:5514-5527.

Zheng Y, Horii A, Appleton I, Darlington CL, Smith PF (2001) Damage to the vestibular inner ear causes long-term changes in neuronal nitric oxide synthase expression in the rat hippocampus. Neuroscience 105:1-5. 